\title{
Three-Family SO(10) Grand Unification in String Theory
}

\author{
Zurab Kakushadze* and S.-H. Henry Tye \\ Newman Laboratory of Nuclear Studies, Cornell University, \\ Ithaca, New York 14853-5001
}

(Received 30 May 1996)

\begin{abstract}
The construction of a supersymmetric $\mathrm{SO}(10)$ grand unification with 5 left-handed and 2 right-handed families in the four-dimensional heterotic string theory is presented. The model has one $\mathrm{SO}(10)$ adjoint Higgs field. The SO(10) current algebra is realized at level 3. [S0031-9007(96)00948-9]

PACS numbers: 11.25.Mj, 12.10.Dm, 12.60.Jv
\end{abstract}

The outstanding question of superstring theory is how does it describe our Universe. The space of classical supersymmetric string vacua has a large degeneracy, which may be described by a set of parameters, or moduli. We expect string dynamics to lift, partially or completely, this huge degeneracy in the moduli space. Each point in this moduli space corresponds to a particular string model. To be specific, let us consider the heterotic superstring case. Ignoring the possibility of enhanced gauge symmetry from nonperturbative effects, the rank of the gauge group is 22 or less [for our counting purpose, U(1) has rank 1]. After accommodating the standard model of strong and electroweak interactions (with minimum rank 4), there is still plenty of room (i.e., with maximum rank 18) for a large hidden sector. The possible choices of the hidden sector are myriad and largely unexplored, and the dynamics in each case is very complicated. The difficulty of string phenomenology is the lack of an objective criteria that would select a particular model among the numerous possibilities; that is, the moduli space of the hidden sector is too big for the string dynamics to be analyzed systematically. This difficulty may be solved by considering grand unification in string theory. As we shall see, after imposing some rather simple phenomenological constraints, the hidden sector seems to be unique and the remaining moduli space is essentially reduced to a one-dimensional space. The string dynamics in this case should be within reach.

The apparent unification of the gauge couplings in the context of supersymmetry when extrapolated to high energy scales has created a lot of interest in supersymmetric grand unified theories. To realize a grand unified model in the superstring theory, there is a very stringent constraint. It is well known that, in field theory, adjoint Higgs (or other appropriate higher-dimensional) representation is necessary for a grand unified gauge group to break spontaneously to the $\mathrm{SU}(3) \otimes \mathrm{SU}(2) \otimes \mathrm{U}(1)$ gauge group of the standard model. It is also known that, for current algebras at level 1, space-time supersymmetry with chiral fermions do not coexist with massless scalar fields in the adjoint or higher-dimensional representations of the gauge group in heterotic string models. From these facts, one concludes that a grand unified model in the superstring theory is pos- sible only if the grand unified gauge group comes from current algebras at levels higher than 1 .

Grand unified models in the superstring theory are sometimes referred to as grand unified string theories (GUST). The first GUST analysis was given by Lewellen [1]. In particular, he constructed an SO(10) GUST with four chiral families. Next, Schwartz extended the construction to include an SU(5) GUST, also with four chiral families [2]. More recently, Erler used the orbifold method [3] to construct $E_{6}$ GUSTs, again with an even number of families [4]. In the mean time, there are a number of other interesting related works [5]. Since nature seems to have only three light families, attempts were made to construct a GUST with three chiral families, so far, unsuccessful. This suggests that a 3-family GUST either does not exist, or, more interestingly, is extremely limited. The GUST models with even number of families mentioned above all involve a level-2 gauge group. So, to find a GUST with three families, it is natural to go to level-3 gauge groups. In this work, we shall report the construction of such a model: An $N=1$ supersymmetric SO(10) GUST with an adjoint Higgs and three chiral families; to be more precise, the model has five lefthanded and two right-handed chiral families.

The model has the gauge group $\mathrm{SU}(2)_{1} \otimes \mathrm{U}(1) \otimes M \otimes$ $\mathrm{SO}(10)_{3} \otimes \mathrm{U}(1)$, where the subscripts indicate the level of the current algebra. Here $M$ can be $\mathrm{SU}(2)_{3} \otimes \mathrm{U}(1)$, $\mathrm{U}(1)^{2}, \mathrm{U}(1)$, or empty. There is only one adjoint Higgs representation in the $\mathrm{SO}(10)$. Each of the five $\mathbf{1 6}_{L}$ and the two $\mathbf{1 6}_{R}$ families of $\mathrm{SO}(10)$ is accompanied by a $\mathbf{1 0}$ and a singlet of $\mathrm{SO}(10)$. They have quantum numbers in $\mathrm{U}(1)^{2} \otimes M$, but are singlets under the $\mathrm{SU}(2)_{1}$. So, by definition, the $\mathrm{SU}(2)_{1}$ gauge group is the hidden sector, while $M$ may be considered as a horizontal symmetry. There are massless supermultiplets that form doublets in this hidden $\mathrm{SU}(2)_{1}$. They are singlets under $\mathrm{SO}(10)$ and neutral under U(1). However, these doublets, as well as the chiral families of $\mathrm{SO}(10)$, have nontrivial quantum values in $M$. So we may also consider $M$ as the gauge group for the messenger or mediator sector, linking the hidden and the visible sectors.

Phenomenologically, one wants a hidden sector that will become strong so that supersymmetry may be dynamically 
broken. The gauge coupling of a given group $G$ in the model at a scale $\mu$ below the string scale $m_{s}$ is related to it via

$$
1 / \alpha_{G}(\mu)=k_{G} / \alpha_{\text {string }}+\left(b_{0} / 4 \pi\right) \ln \left(m_{s}^{2} / \mu^{2}\right),
$$

where $k_{G}$ is the level of the gauge group. For a U(1) gauge theory, $k=2 r^{2}$ if the U(1) charge is normalized so that the lowest allowed value is \pm 1 (with conformal highest weight $r^{2} / 2$ ), and $r$ is the compactification radius of the corresponding world-sheet boson. The constant $b_{0}$ is the one-loop coefficient of the beta function. The hidden sector $\mathrm{SU}(2)_{1}$ is asymptotically free while $M$ is not. At the string scale, the hidden $\mathrm{SU}(2)_{1}$ coupling $\alpha_{2}$ is 3 times that of the $\mathrm{SO}(10)_{3}$. So, for typical values of the $\mathrm{SO}(10)$ grand unification coupling, $\alpha_{2}$ becomes large at a rather low scale, within a few orders of magnitude above the electroweak scale. If $M$ is empty, the hidden sector physics will have negligible impact on the physics in the visible sector. Since this is phenomenologically undesirable, we shall demand a nonempty $M$ in the 3-family GUST.

In Table I, we give the massless spectra for two of these cases. The first model is $\mathrm{SU}(2)_{1} \otimes \mathrm{SU}(2)_{3} \otimes$ $\mathrm{SO}(10)_{3} \otimes \mathrm{U}(1)^{3}$, with the $\mathrm{U}(1)$ charges normalized to radii $(1 / \sqrt{6}, 1 / 3 \sqrt{2}, 1 / 6)$. The second model is $\mathrm{SU}(2)_{1} \otimes$ $S O(10)_{3} \otimes \mathrm{U}(1)^{4}$, with the $\mathrm{U}(1)$ charges normalized to radii $(1 / \sqrt{6}, 1 / 6 \sqrt{2}, 1 / 6 \sqrt{2}, 1 / 6)$. It is easy to check that both models are anomaly free.

TABLE I. The massless spectra of the two models SU $(2)^{2} \otimes$ $\mathrm{SO}(10) \otimes \mathrm{U}(1)^{3}$ and $\mathrm{SU}(2) \otimes \mathrm{SO}(10) \otimes \mathrm{U}(1)^{4}$. The gravity, dilaton, and gauge supermultiplets are not shown.

\begin{tabular}{ccc}
\hline \hline $\mathrm{M}$ & $\mathrm{SU}(2)_{3} \otimes \mathrm{U}(1)$ & $\mathrm{U}(1) \otimes \mathrm{U}(1)$ \\
\hline & $(\mathbf{1}, \mathbf{1}, \mathbf{4 5})(0,0,0)$ & $(\mathbf{1}, \mathbf{4 5})(0,0,0,0)$ \\
& $(\mathbf{1}, \mathbf{3}, \mathbf{1})(0,0,0)$ & $2(\mathbf{1}, \mathbf{1})(0,+12,0,0)_{L}$ \\
$\mathrm{U}$ & $(\mathbf{1}, \mathbf{1}, \mathbf{1})(0,-6,0)_{L}$ & $2(\mathbf{1}, \mathbf{1})(0,0,+12,0)_{L}$ \\
& $2(\mathbf{1}, \mathbf{4}, \mathbf{1})(0,+3,0)_{L}$ & $3(\mathbf{1}, \mathbf{1})(0,-6,0,0)_{L}$ \\
& $2(\mathbf{1}, \mathbf{2}, \mathbf{1})(0,-3,0)_{L}$ & $3(\mathbf{1}, \mathbf{1})(0,0,-6,0)_{L}$ \\
& $2(\mathbf{1}, \mathbf{2}, \mathbf{1 6})(0,-1,-1)_{L}$ & $2(\mathbf{1}, \mathbf{1 6})(0,+2,+2,-1)_{L}$ \\
& $2(\mathbf{1}, \mathbf{2}, \mathbf{1 0})(0,-1,+2)_{L}$ & $2(\mathbf{1}, \mathbf{1 0})(0,+2,+2,+2)_{L}$ \\
& $2(\mathbf{1}, \mathbf{2}, \mathbf{1})(0,-1,-4)_{L}$ & $2(\mathbf{1}, \mathbf{1})(0,+2,+2,-4)_{L}$ \\
& $(\mathbf{1}, \mathbf{1}, \mathbf{1 6})(0,+2,-1)_{L}$ & $(\mathbf{1}, \mathbf{1 6})(0,-4,-4,-1)_{L}$ \\
& $(\mathbf{1}, \mathbf{1}, \mathbf{1 0})(0,+2,+2)_{L}$ & $(\mathbf{1}, \mathbf{1 0})(0,-4,-4,+2)_{L}$ \\
$\mathrm{~T}$ & $(\mathbf{1}, \mathbf{1}, \mathbf{1})(0,+2,-4)_{L}$ & $(\mathbf{1}, \mathbf{1})(0,-4,-4,-4)_{L}$ \\
3 & & $(\mathbf{1}, \mathbf{1 6})(0,-4,+2,-1)_{L}$ \\
& & $(\mathbf{1}, \mathbf{1 0})(0,-4,+2,+2)_{L}$ \\
& & $(\mathbf{1}, \mathbf{1})(0,-4,+2,-4)_{L}$ \\
& & $(\mathbf{1}, \mathbf{1 6})(0,+2,-4,-1)_{L}$ \\
& & $(\mathbf{1}, \mathbf{1 0})(0,+2,-4,+2)_{L}$ \\
& & $(\mathbf{1}, \mathbf{1})(0,+2,-4,-4)_{L}$ \\
$\mathrm{~T}$ & $(\mathbf{1}, \mathbf{1}, \overline{\mathbf{1 6}})( \pm 1,+1,+1)_{L}$ & $(\mathbf{1}, \overline{\mathbf{1 0}})( \pm 1,+1,+1,+1)_{L}$ \\
6 & $(\mathbf{1}, \mathbf{1}, \mathbf{1 0})( \pm 1,+1,-2)_{L}$ & $(\mathbf{1}, \mathbf{1 0})( \pm 1,+1,+1,-2)_{L}$ \\
& $(\mathbf{1}, \mathbf{1}, \mathbf{1})( \pm 1,+1,+4)_{L}$ & $(\mathbf{1}, \mathbf{1})( \pm 1,+1,+1,+4)_{L}$ \\
& $(\mathbf{2}, \mathbf{2}, \mathbf{1})(0,0,0)_{L}$ & $2(\mathbf{2}, \mathbf{1})(0,-3,-3,0)_{L}$ \\
$\mathrm{~T}$ & $(\mathbf{2}, \mathbf{4}, \mathbf{1})(0,0,0)_{L}$ & $(\mathbf{2}, \mathbf{1})(0, \pm 9,+3,0)_{L}$ \\
2 & & $(\mathbf{2}, \mathbf{1})(0,+3, \pm 9,0)_{L}$ \\
& $(\mathbf{1}, \mathbf{1}, \mathbf{1})( \pm 3,-3,0)_{L}$ & $(\mathbf{1}, \mathbf{1})( \pm 3,-3,-3,0)_{L}$ \\
\hline \hline
\end{tabular}

The spectrum of the $M=\mathrm{U}(1)$ model can be obtained in the same way as for the other two models, which we will discuss in a moment. Since these models are connected by flat moduli, one may take an effective field theory approach to obtain the massless spectrum of the $M=\mathrm{U}(1)$ model. Give one of the $\mathrm{SU}(2)_{3}$ doublets in the $U$ sector of the $M=\mathrm{SU}(2)_{3} \otimes \mathrm{U}(1)$ (radius $1 / 3 \sqrt{2}$ ) model a nonzero expectation value, and the gauge group $\mathrm{SU}(2)_{3} \otimes \mathrm{U}(1)$ breaks to $\mathrm{U}(1)$, with the assignment of this $\mathrm{U}(1)$ charge completely determined. In the field theoretic approach, we may also use the $\mathrm{SO}(10)$ adjoint Higgs fields to break $\mathrm{SO}(10)$ to $\mathrm{SU}(5)$ (with its own adjoint Higgs fields).

Let us turn to the construction of the models, which is carried out in the orbifold framework [3]. The construction is achieved by turning on Wilson lines in the $\mathrm{SO}(32)$ model toroidally compactified to four dimensions, followed by a $\mathbf{Z}_{6}$ orbifold. To make the discussion easier to follow, we split the $\mathbf{Z}_{6}$ twist into a $\mathbf{Z}_{3}$ twist followed by a $\mathbf{Z}_{2}$ twist. A more detailed discussion will appear separately [6], where other GUSTs are also discussed.

Our starting point is an $N=4$ space-time supersymmetric Narain model [7] with the momenta of the internal bosons spanning an even self-dual Lorentzian lattice $\Gamma^{6,22}=\Gamma^{2,2} \otimes \Gamma^{4,4} \otimes \Gamma^{16}$, where each factor is even self dual. Here $\Gamma^{2,2}=\left\{\left(p_{R} \| p_{L}\right)\right\}$, with $p_{R}, p_{L} \in \tilde{\Gamma}^{2}[\mathrm{SU}(3)$ weight lattice], and $p_{L}-p_{R} \in \Gamma^{2}$ [SU(3) root lattice]. Note that $\Gamma^{2}=\left\{e_{i} n^{i}\right\}$ and $\tilde{\Gamma}^{2}=\left\{\tilde{e}^{i} m_{i}\right\}$, where $e_{i}$ are the SU(3) simple roots, and their duals $\tilde{e}^{i}$ are the corresponding weight vectors (i.e., $e_{i} \cdot \tilde{e}^{j}=\delta_{i}^{j}, i, j=1,2$ ). $\Gamma^{16}$ is the self-dual $\operatorname{Spin}(32) / \mathbf{Z}_{2}$ lattice. $\Gamma^{4,4}$ is an even self-dual Lorentzian lattice that admits a symmetric $\mathbf{Z}_{3}$ orbifold, such that both complex coordinates are simultaneously twisted. The most general $\Gamma^{4,4}$ that possesses such a $\mathbf{Z}_{3}$ symmetry has an 8 -dimensional moduli space and a generic gauge group $R=\mathrm{U}(1)^{4}$. After orbifolding, the resulting $M$ is empty in this generic case. To obtain a nonempty $M$, we restrict ourselves to a special one-dimensional subspace of the moduli space, which has an enhanced $R$. Recall that $\Gamma^{4,4}$ is a momentum lattice corresponding to a compactification on a torus defined by $X_{I}=X_{I}+E_{I}$. In our case, the vectors $E_{I}$ (and their duals $\tilde{E}^{I}$ ) can be expressed in terms of the SU(3) root and weight vectors $e_{i}$ and $\tilde{e}^{i}$ :

$$
\begin{aligned}
& E_{1}=\left(e_{1}, 0\right), \quad E_{2}=\left(e_{2}, 0\right), \\
& E_{3}=\left(-h \tilde{e}^{2}, g e_{1}\right), \quad E_{4}=\left(h \tilde{e}^{1}, g e_{2}\right),
\end{aligned}
$$

where $g \equiv \sqrt{1-h^{2} / 3}$. For $0<h<1$, and with appropriate constant antisymmetric background fields, we have an enhanced gauge group $R=\mathrm{SU}(3) \otimes \mathrm{U}(1)^{2}$. At special points $h=0,1, \Gamma^{4,4}$ can be generated by $\left(0 \| E_{I}\right)$ and $\left(\tilde{E}^{I} \| \tilde{E}^{I}\right)$, and the gauge symmetry is enhanced to $R=$ $\mathrm{SU}(3)^{2}$ and $R=S O(8)$, respectively. As we shall see, these three cases correspond to $M=\mathrm{U}(1), M=\mathrm{U}(1)^{2}$, and $M=\mathrm{SU}(2)_{3} \otimes \mathrm{U}(1)$, respectively. 
Next we turn on Wilson lines that break the $S O(32)$ subgroup to $S O(10)^{3} \otimes S O(2)$. This must be done in a way so that the resulting Narain model, which we will refer to as $N 1$, still possesses the $\mathbf{Z}_{3}$ symmetry of the space part of the $N 0$ model (so that the $\mathbf{Z}_{3}$ orbifolding performed in the next step is possible), and also, the three $\mathrm{SO}(10)$ s must be symmetric under a $\mathbf{Z}_{3}$ permutation [so that modding out by this outer automorphism will yield $\left.\mathrm{SO}(10)_{3}\right]$. The above requirements (up to equivalent representations) fix the Wilson lines to have the following form:

$$
\begin{aligned}
& U_{1}=\left(e_{1} / 2 \| 0\right)\left(P_{R}^{(1)} / 2|| P_{L}^{(1)} / 2\right)(\mathbf{s}|\mathbf{0}| \mathbf{0} \mid \bar{S}), \\
& U_{2}=\left(e_{2} / 2 \| 0\right)\left(P_{R}^{(2)} / 2 \| P_{L}^{(2)} / 2\right)(\mathbf{0}|\mathbf{s}| \mathbf{0} \mid \bar{S}) .
\end{aligned}
$$

Here we are writing the Wilson lines as shift vectors in the $\Gamma^{6,22}$ lattice. Thus $U_{1}$ and $U_{2}$ are order $2\left(\mathbf{Z}_{2}\right)$ shifts. Here $e_{1} / 2$ and $e_{2} / 2$ are the right-moving shifts in $\Gamma^{2,2}$. The $\Gamma^{4,4}$ shifts are given by $P_{R}^{(1)}=P_{L}^{(1)}+E_{1}+E_{3}$, $P_{L}^{(1)}=-h \tilde{E}^{4}$ and $P_{R}^{(2)}=P_{L}^{(2)}+E_{2}+E_{4}, P_{L}^{(2)}=h \tilde{E}^{3}$. The $\mathrm{SO}(32)$ shifts are given in the $\mathrm{SO}(10)^{3} \otimes \mathrm{SO}(2)$ basis. In this basis, $\mathbf{0}$ stands for the null vector, $\mathbf{v}$ $(V)$ is the vector weight, whereas $\mathbf{s}(S)$ and $\overline{\mathbf{s}}(\bar{S})$ are the spinor and antispinor weights of $\mathrm{SO}(10)$ [SO(2)]. [For $\mathrm{SO}(2), V=1, S=1 / 2$, and $\bar{S}=-1 / 2$.] These Wilson lines break the gauge symmetry to $\mathrm{SU}(3) \otimes R \otimes$ $\mathrm{SO}(10)^{3} \otimes \mathrm{SO}(2)$. Note that $R$ is not affected. All the gauge bosons come from the unshifted sector, whereas the shifted sectors give rise to massive states only. Note that, for each twist and/or shift in the model building, we have implicitly chosen the spin structures of the rightmoving world-sheet fermions to be compatible with the world-sheet supersymmetry.

Now we introduce the following $\mathbf{Z}_{3}$ twist on the $N 1$ model:

$$
(\theta \| 0)(\Theta \| \Theta)(\mathcal{P} \mid 2 / 3),
$$

where $\theta$ is a $\mathbf{Z}_{3}$ twist (that is, a $2 \pi / 3$ rotation) that acts only on the right-moving part of the $\Gamma^{2,2}$ lattice (and the corresponding oscillator excitations), and the leftmoving part is untouched. This is an asymmetric orbifold. The $\Gamma^{4,4}$ lattice is twisted symmetrically by the $\mathbf{Z}_{3} \otimes \mathbf{Z}_{3} \Theta$ twist. The three $\mathrm{SO}(10)$ s are permuted by the action of the $\mathbf{Z}_{3}$ outer automorphism twist $\mathcal{P}: \phi_{1}^{I} \rightarrow$ $\phi_{2}^{I} \rightarrow \phi_{3}^{I} \rightarrow \phi_{1}^{I}$, where the real bosons $\phi_{p}^{I}, I=1, \ldots, 5$, correspond to the $p$ th $\mathrm{SO}(10)$ subgroup, $p=1,2,3$. We can define new bosons $\varphi^{I} \equiv \frac{1}{\sqrt{3}}\left(\phi_{1}^{I}+\phi_{2}^{I}+\phi_{3}^{I}\right)$; the other ten real bosons are complexified via linear combinations $\Phi^{I} \equiv \frac{1}{\sqrt{3}}\left(\phi_{1}^{I}+\omega \phi_{2}^{I}+\omega^{2} \phi_{3}^{I}\right)$ and $\left(\Phi^{I}\right)^{\dagger} \equiv$ $\frac{1}{\sqrt{3}}\left(\phi_{1}^{I}+\omega^{2} \phi_{2}^{I}+\omega \phi_{3}^{I}\right)$, where $\omega=\exp (2 \pi i / 3)$. Un$\operatorname{der} \mathcal{P}, \varphi^{I}$ is invariant, while $\Phi^{I}\left[\left(\Phi^{I}\right)^{\dagger}\right]$ are eigenstates with eigenvalue $\omega^{2}(\omega)$, i.e., modded out. The $\mathbf{Z}_{3}$ invariant states form irreducible representations (irreps) of $\mathrm{SO}(10)_{3}$. Finally, string consistency requires the inclusion of the $2 / 3$ shift in the $\mathrm{SO}(2)$ lattice.
The model (which we will refer to as $A 1$ ) that results from twisting by the above $\mathbf{Z}_{3}$ twist has $N=1$ space-time supersymmetry. First, we discuss the untwisted sector of this model. All the gauge bosons come from the untwisted sector, and the gauge group is $\mathrm{SU}(3)_{1} \otimes R_{1} \otimes$ $\mathrm{SO}(10)_{3} \otimes \mathrm{U}(1)$, where $R_{1} \subset R$ depends on the value of the modulus $h$. At the generic point $0<h<1$, $R_{1}=\mathrm{U}(1)^{2}$. At $h=0, R_{1}$ is enhanced to $\mathrm{U}(1)^{4}$, and for $h=1, R_{1}=\mathrm{SU}(3)_{3}$. The latter case corresponds to a special breaking $\mathrm{SO}(8)_{1} \supset \mathrm{SU}(3)_{3}$ that results from the $\Theta$ twist. This can be understood as a $\mathbf{Z}_{3}$ twist in the $\mathrm{SU}(3)_{1}$ subgroup of $\mathrm{SO}(8)_{1} \supset \mathrm{SU}(3)_{1} \otimes \mathrm{U}(1)^{2}$ accompanied by a $\mathbf{Z}_{3}$ twist on the $\mathbf{Z}_{3}$ symmetry in the $\mathrm{U}(1)^{2}$ subgroup, the latter simply being the triality symmetry of the $\mathrm{SO}(8)_{1}$ Dynkin diagram under which $8_{v} \rightarrow 8_{s} \rightarrow 8_{c} \rightarrow$ $8_{v}$. Besides the gauge supermultiplets, other massless states appearing in the untwisted sector are three copies of adjoint Higgs fields of $\mathrm{SO}(10)_{3}$. There are also three copies of massless states in irreps of $R_{1}$. For example, at $h=1$, we have three copies of chiral fermions in $\mathbf{1 0}_{L}$ of $\mathrm{SU}(3)_{3}$ (here we define these states to be left handed).

The twisted sectors give rise to chiral matter fields of $\mathrm{SO}(10)_{3}$. The asymmetric $\mathbf{Z}_{3}$ twist $(\theta \| 0)$ in $\Gamma^{2,2}$ contributes only a factor of 1 to the number of fixed points as the factor 3 contributed by the right movers is canceled against the volume factor of the invariant sublattice, which is $\Gamma^{2}$. Similarly, the outer automorphism twist contributes only one fixed point. This follows from the form of the invariant sublattice, which is $\Gamma^{6}=\{(\sqrt{3} \mathbf{q} \mid Q)\}$, where $(\mathbf{q} \mid Q)=(\mathbf{0} \mid 0),(\mathbf{v} \mid V),(\mathbf{s} \mid S),(\overline{\mathbf{s}} \mid \bar{S})$. The momenta in the twisted and inverse twisted sector belong to the shifted dual lattices $\tilde{\Gamma}^{6}+(\mathbf{0} \mid \pm 2 / 3)$, respectively, where $\tilde{\Gamma}^{6}=$ $\{(\mathbf{q} / \sqrt{3} \mid Q)\}$. The only nontrivial contribution to the number of fixed points in the twisted sectors comes from the symmetric $\mathbf{Z}_{3}$ twist $(\Theta \| \Theta)$ in $\Gamma^{4,4}$. This twist contributes $9=3_{R} \times 3_{L}$ fixed points. So there are nine fixed points in the twisted sector. The left-moving fixed points fall under irreps of the $R_{1}$ group. For $R_{1}=\mathrm{SU}(3)_{3}$ we have three copies (due to the three right-moving fixed points) of massless states in the $\mathrm{SU}(3)_{1} \otimes \mathrm{SU}(3)_{3} \otimes \mathrm{SO}(10)_{3}$ irreps $(\mathbf{1}, \overline{\mathbf{3}}, \mathbf{1 6})(-1)_{L},(\mathbf{1}, \overline{\mathbf{3}}, \mathbf{1 0})(+2)_{L},(\mathbf{1}, \overline{\mathbf{3}}, \mathbf{1})(-4)_{L}$. [Here we give the $\mathrm{U}(1)$ charge in parentheses, and its normalization is $1 / 6$.] Note that the $\mathrm{SU}(3)_{3}$ chiral anomaly in the twisted sectors is canceled by that in the untwisted sector as a $\mathbf{1 0}_{L}$ of $\mathrm{SU}(3)_{3}$ has 27 times the anomaly contribution of a $\mathbf{3}_{L}$. The model is also $\mathrm{U}(1)$ anomaly free due to the underlying $E_{6}$ structure of the $\mathrm{SO}(10)_{3} \otimes$ $\mathrm{U}(1)$ matter fields as can be seen from the branching $27=\mathbf{1 6}(-1)+\mathbf{1 0}(+2)+\mathbf{1}(-4)$ under the breaking $E_{6} \supset \mathrm{SO}(10) \otimes \mathrm{U}(1)$.

To obtain the final model, let us orbifold the $A 1$ model by the following symmetric $\mathbf{Z}_{2}$ twist:

$$
\left(0|| e_{1} / 2\right)(-\mathbf{1}||-\mathbf{1})\left(0^{15} \mid 0\right) \text {. }
$$

Here the left-moving momenta of $\Gamma^{2,2}$ are shifted by $e_{1} / 2$, while $\Gamma^{16}$ is untouched. $\Gamma^{4,4}$ is symmetrically twisted by 
a diagonal $\mathbf{Z}_{2}$ twist ( $\mathbf{1}$ is a $4 \times 4$ identity matrix). This $\mathbf{Z}_{2}$ orbifold preserves the $N=1$ supersymmetry.

First, we discuss the untwisted sector U. All the gauge bosons still come from the untwisted sector, and the gauge group is now $\mathrm{SU}(2)_{1} \otimes \mathrm{U}(1) \otimes M \otimes \mathrm{SO}(10)_{3} \otimes$ $\mathrm{U}(1)$. The $\mathrm{SU}(2)_{1} \otimes \mathrm{U}(1)$ factor emerges from the regular breaking $\mathrm{SU}(3)_{1} \supset \mathrm{SU}(2)_{1} \otimes \mathrm{U}(1)$, due to the $e_{1} / 2$ shift. Since this shift is required by string consistency, we see that $\mathrm{SU}(2)_{1}$ is the biggest possible hidden sector in our construction. $M$ is a subgroup of $R_{1}$, depending on the value of $h$. For $0<h<1, M=\mathrm{U}(1)$. For $h=0, M$ is enhanced to $\mathrm{U}(1) \otimes \mathrm{U}(1)$. For $h=1, M$ is enhanced to $\mathrm{SU}(2)_{3} \otimes \mathrm{U}(1)$, which is a result of the regular breaking $\mathrm{SU}(3)_{3} \supset \mathrm{SU}(2)_{3} \otimes \mathrm{U}(1)$. Let us focus on the $h=1$ case, since the other cases are simpler. Note that there are no massless states in the nontrivial irreps of $\mathrm{SU}(2)_{1} \otimes \mathrm{U}(1) \otimes \mathrm{U}(1)$. Two out of the three copies of the massless states in the irreps of $R_{1} \otimes \mathrm{SO}(10)_{3}$ in the $A 1$ model have $\mathbf{Z}_{2}$ phase -1 , whereas the third copy has the phase 1 . Since the adjoint irreps of $\mathrm{SO}(10)_{3}$ are singlets under $R_{1}$, only one copy of the massless $\mathrm{SO}(10)_{3}$ adjoint Higgs fields remains in the final model. We also have one copy of $\mathbf{1}(-6)$ and $\mathbf{3}(0)$ each, and two copies of $2(-3)$ and $\mathbf{4}(+3)$ each [The $U(1)$ charge is normalized to $1 / 3 \sqrt{2}$.] These states arise as a result of the branching [under $\left.\mathrm{SU}(3)_{3} \supset \mathrm{SU}(2)_{3} \otimes \mathrm{U}(1)\right] \mathbf{1 0}=$ $\mathbf{1}(-6)+\mathbf{2}(-3)+\mathbf{3}(0)+\mathbf{4}(+3)$, where the singlet and the triplet have the $\mathbf{Z}_{2}$ phase 1, while the doublet and the quartet have the phase -1 .

Next, let us consider the $\mathbf{Z}_{3}$ twisted (plus its inverse) sector T3. We start with the nine fixed points in this sector. Of these nine fixed points, the one at the origin is invariant under the $\mathbf{Z}_{2}$ twist. The remaining eight fixed points form four pairs, and the $\mathbf{Z}_{2}$ twist permutes the two fixed points in each pair. Forming four symmetric and four antisymmetric combinations, we have $9=5(1)+$ $4(-1)$ (where the $\mathbf{Z}_{2}$ phases are given in parentheses); that is, five of the original nine are invariant under the $\mathbf{Z}_{2}$ twist. Since there is no relative phase between the $T 2$ and $T 3$ sectors, these five copies of the $\mathrm{SO}(10)_{3}$ chiral matter fields survive, while the other four are projected out. These five copies transform in the irreps of $M$. We have two copies of $(\mathbf{1}, \mathbf{2}, \mathbf{1 6})(0,-1,-1)_{L}$ and one copy of $(\mathbf{1}, \mathbf{1}, \mathbf{1 6})(0,+2,-1)_{L}$, plus the corresponding vector and singlet irreps of $\mathrm{SO}(10)_{3}$ [the $\mathrm{U}(1)$ charges are normalized to $(1 / \sqrt{6}, 1 / 3 \sqrt{6}, 1 / 6)]$.

Next, consider the $\mathbf{Z}_{6}$ twisted (plus its inverse) sector T6. The sublattice invariant under the $\mathbf{Z}_{6}$ twist is the same as that for the $\mathbf{Z}_{3}$ twist. The number of fixed points in the $T 6$ sector is one. The massless chiral fields are singlets under $\mathrm{SU}(2)_{1} \otimes \mathrm{SU}(2)_{3}$ : $(\mathbf{1}, \mathbf{1}, \mathbf{1 6})( \pm 1,-1,-1)_{R}$, plus the corresponding vector and singlet irreps of $\mathrm{SO}(10)_{3}$. Note that these states are right handed, and come in pairs $[ \pm 1$ of the first $\mathrm{U}(1)$ charge]. So, effectively, we have a total of $3=5-2$ lefthanded chiral families of 16's.
Last, we consider the $\mathbf{Z}_{2}$ twisted sector $T 2$. Let us consider first the twisted sector of the $\mathbf{Z}_{2}$ orbifold of the $N 1$ model, and then its $\mathbf{Z}_{3}$-invariant states that are present in the final model. The sublattice invariant under the $\mathbf{Z}_{2}$ twist is given by the sublattice of $\Gamma^{2,2} \otimes \Gamma^{16}$ invariant under the Wilson lines $U_{1}$ and $U_{2}$. The metric of this sublattice has determinant 16. Therefore, the number of fixed points is $4_{R} \times 4_{L} / \sqrt{16}=2_{R} \times 2_{L}$. The $\mathbf{Z}_{2}$ orbifold breaks $\mathrm{SU}(3) \otimes \mathrm{SO}(8)$ to $\mathrm{SU}(2) \otimes \mathrm{U}(1) \otimes$ $\mathrm{SU}(2)^{4}$, with two massless sets of $(\mathbf{2}, \mathbf{1 , 2 , 2 , 2 ) ( 0 )}$ and $(\mathbf{1}, \mathbf{2}, \mathbf{1}, \mathbf{1}, \mathbf{1})( \pm 3)$. Now consider the action of the $\mathbf{Z}_{3}$ twist. It converts the last three $\mathrm{SU}(2)$ to $\mathrm{SU}(2)_{3}$, while breaking the second $\mathrm{SU}(2)$ to $\mathrm{U}(1)$. The resulting $\mathbf{Z}_{3^{-}}$ invariant massless states are $(\mathbf{2}, \mathbf{2})$ and $(\mathbf{2}, \mathbf{4})$ [in $\mathrm{SU}(2) \otimes$ $\mathrm{SU}(2)_{3}$ ] plus a pair of singlets. All the states in nontrivial irreps of $\mathrm{SO}(10)_{3} \otimes \mathrm{U}(1)$ are massive. This concludes our construction.

We have explored various combinations of $\mathbf{Z}_{3}$ twists and shifts, but failed to obtain the 3-family feature. This leads us to the additional $\mathbf{Z}_{2}$ twist used above. Within this framework, we have also obtained a variation of the above $M=\mathrm{SU}(2)_{3} \otimes \mathrm{U}(1)$ model; the only difference is the assignment of the $M$ quantum numbers and $\mathrm{U}(1)$ charges. These points and other GUSTs will be discussed in Ref. [6]. In conclusion, we see that the realization of the 3-family grand unification in string theory imposes very powerful constraints in the moduli space.

We thank Paul Aspinwall and Gary Shiu for discussions. This work is supported in part by the National Science Foundation.

*Address after September 1, 1996: Lyman Laboratory, Harvard University, Cambridge, MA 02138. Electronic address: zurab@hepth.cornell.edu

[1] D. C. Lewellen, Nucl. Phys. B 337, 61 (1990).

[2] J. A. Schwartz, Phys. Rev. D 42, 1777 (1990).

[3] L. Dixon, J. Harvey, C. Vafa, and E. Witten, Nucl. Phys. B 261, 620 (1985); 274, 285 (1986); K. S. Narain, M.H. Sarmadi, and C. Vafa, Nucl. Phys. B 288, 551 (1987).

[4] J. Erler, Report No. SCIPP 96/10, hep-th/9602032.

[5] A. Font, L.E. Ibáñez, and F. Quevedo, Nucl. Phys. B 345, 389 (1990); S. Chaudhuri, S.-W. Chung, G. Hockney, and J.D. Lykken, Nucl. Phys. B 456, 89 (1995); G. B. Cleaver, Nucl. Phys. B 456, 219 (1995); A. Aldazabal, A. Font, L. E. Ibáñez, and A. M. Uranga, Nucl. Phys. B 452, 3 (1995); Report No. hep-th/9508033. See also L.E. Ibáñez, H. P. Nilles, and F. Quevedo, Nucl. Phys. B 307, 109 (1988).

[6] Z. Kakushadze and S.-H.H, Tye, Cornell Report No. CLNS-96/1413, hep-th/9607138.

[7] K. S. Narain, Phys. Lett. B 169, 41 (1986); K. S. Narain, M. H. Sarmadi, and E. Witten, Nucl. Phys. B 279, 369 (1987). 